Drug utilization according to reason for prescribing: a pharmacoepidemiologic method based on an indication hierarchy

Kildemoes, Helle Wallach; Hendriksen, Carsten; Morten, Andersen

Published in:

Pharmacoepidemiology and Drug Safety

DOI:

$10.1002 /$ pds. 2195

Publication date:

2011

Document version

Early version, also known as pre-print

Citation for published version (APA):

Kildemoes, H. W., Hendriksen, C., \& Morten, A. (2011). Drug utilization according to reason for prescribing: a pharmacoepidemiologic method based on an indication hierarchy. Pharmacoepidemiology and Drug Safety.

https://doi.org/10.1002/pds.2195 


\title{
Drug utilization according to reason for prescribing: a pharmacoepidemiologic method based on an indication hierarchy
}

\author{
Helle Wallach Kildemoes ${ }^{1,2 *}$, Carsten Hendriksen ${ }^{1}$ and Morten Andersen ${ }^{2,3}$ \\ ${ }^{1}$ Centre for Healthy Aging, Department of Public Health, University of Copenhagen, Copenhagen, Denmark \\ ${ }^{2}$ Research Unit for General Practice, University of Southern Denmark, Odense, Denmark \\ ${ }^{3}$ Centre for Pharmacoepidemiology, Karolinska Institutet, Stockholm, Sweden
}

\begin{abstract}
Purpose To develop a pharmacoepidemiologic method for drug utilization analysis according to indication, gender, and age by means of register-based information. Statin utilization in 2005 was applied as an example.

Methods Following the recommendations for statin therapy, we constructed an indication hierarchy with eight mutually exclusive levels of register markers of cardiovascular disease and diabetes. Danish residents, as of January 1, 1996, were followed at the individual level in nationwide registers with respect to dispensed prescriptions of cardiovascular drugs and antidiabetics (1996-2005) along with discharge diagnoses and surgical procedures (1977-2005). The highest current possible indication level was assigned to all cohort members. Stratified by indication, gender, and age, statin treatment prevalence and incidence were calculated.

Results Statin treatment prevalence was highest among individuals with myocardial infarction and tended to be higher among men with indications in the upper part of the hierarchy, but it was higher among women (especially the elderly) in the lower part of the hierarchy. Treatment incidence rates followed roughly the same pattern. Women with no register marker or primary hypertension accounted for almost $50 \%$ of all incident female users; among men, the figure was 35\%. The proportion of incident users with ischemic heart disease or myocardial infarction increased with age.

Conclusion The proposed indication hierarchy provided new insight into prescription patterns of statins. The method can be implemented for other drug categories and could be useful for studying trends in drug utilization, differential drug adherence, and cross-national comparisons. Copyright (C) 2011 John Wiley \& Sons, Ltd.
\end{abstract}

KEY WORDS - drug utilization; prescribing indication; indication hierarchy; pharmacoepidemiologic method; statins

Abbreviations: CVD, cardiovascular disease; MI, myocardial infarction; IHD, ischemic heart disease; PAD, peripheral arterial disease in the lower limbs; PAC, potential atherosclerotic conditions; DNPR, Danish National Prescription Registry; ATC, Anatomical Therapeutic Chemical classification system; DDD, defined daily dose

Received 27 December 2010; Revised 18 May 2011; Accepted 24 May 2011

\section{BACKGROUND}

During recent decades, the utilization of many drug categories, such as lipid-lowering drugs, antihypertensives, antidepressants, and bisphosphonates, has increased dramatically. A major reason for this increase could be the widening of indications for prescribing, following the evidence of beneficial

*Correspondence to: H. Wallach Kildemoes, Section for Health Services Research, Department of Public Health, University of Copenhagen, CSS, Øster Farimagsgade 5, 1014 Copenhagen, Denmark. E-mail: hewk@sund.ku.dk effects in patients other than those for which the drug originally was intended. For example, the recommendations for prescribing statins (HMG-CoA reductase inhibitors) have broadened from hypercholesterolemia among middle-aged men with myocardial infarction (MI) to a preventive treatment for all individuals at high risk of cardiovascular events regardless of lipid levels ${ }^{1-8}$ and irrespective of age and gender ${ }^{9,10}$.

Although a range of studies from different countries have explored trends in statin prescribing according to age and gender, and some according to broad categories of underlying medical conditions ${ }^{11-17}$, none have applied a consistent pharmacoepidemiologic approach 
to analyze drug utilization according to indication. Thus, it is unknown to what extent an indication creep (i.e., the slow and silent widening of official and unofficial indications, leading to expanding consumption) affects age- and gender-specific drug treatment prevalence and incidence. Knowledge of actual patterns of drug utilization according to indication could help to explain drivers behind the growing drug use. It could also determine to what extent a particular drug is used for its main indication, where the beneficial effect normally is highest and the adverse effectto-benefit ratio presumably is at its lowest.

The nationwide individual-level registries in Denmark include a range of health care and sociodemographic information, representing a unique data source for drug utilization analysis ${ }^{18,19}$. Using the prescription registry, we previously developed a pharmacoepidemiologic semi-Markov model for analyzing the dynamics behind the increasing prevalence of treatment with cardiovascular drugs 20 . By means of the model, we demonstrated that population aging accounted for about $7 \%$ of the growth in treatment prevalence with statins during 1996-2005, whereas increasing treatment incidence accounted for $72 \%$. It is likely that part of the latter increase is explained by a widening of indications and changing prescribing behavior. However, the prescription data contain no structured information about indication. Instead, discharge diagnoses combined with dispensing information for certain drugs may serve as markers of medical conditions present at specific points in time. These register markers can be applied as a proxy for the indication if identifying diagnoses and medication that correspond to the recommended indications for prescribing a particular drug.

The aim of the present study was to develop a pharmacoepidemiologic method to analyze drug utilization according to indication, age, gender, and calendar year, applying a hierarchy of register markers for recommended prescribing indications. We demonstrate the method with statin utilization as an example.

\section{MATERIAL AND METHODS}

The indications for statin therapy cover the range of medical conditions for which lipid-lowering therapy can be recommended based on scientific evidence. Thus, we reviewed the most important randomized placebo-controlled trials on the beneficial effect of statins (i.e., reduced cardiovascular morbidity and mortality) ${ }^{1,3-10}$ and the regularly updated European and Danish national guidelines for prevention of cardiovascular disease (CVD) ${ }^{21-29}$.
Irrespective of age and gender, statins are now recommended for individuals with established atherosclerotic CVD: individuals with MI, ischemic heart disease (IHD), stroke, and atherosclerotic CVD in general, but especially peripheral arterial disease in the lower limbs (PAD) ${ }^{25}$. In addition, statins are recommended for individuals without established CVD, but who are at high risk of CVD, such as individuals with diabetes, asymptomatic individuals with hypertension, or individuals with other risk factors for CVD (i.e., hypercholesterolemia). Preventive drug therapy (statins and/or antihypertensives) is now recommended for asymptomatic individuals with a 10 -year risk of a fatal CVD above 5\%, estimated by means of, for example, the European Systematic Coronary Risk Evaluation 30 . Statins are a priori recommended for individuals with a familial disposition for CVD, such as familial hypercholesterolemia ${ }^{22,23,27,28}$.

Corresponding to the priorities described in guidelines, we constructed a statin indication hierarchy: MI, IHD, stroke, PAD, other potential atherosclerotic conditions (PAC), diabetes, primary hypertension, and a group with no markers of diagnosis (Table 1). The statin indication for a person with several qualifying medical conditions was assumed to be the one at the highest level. Individual-level register information on discharge diagnoses, invasive procedures, and information on drug dispensing were applied as markers of disease prior to initiation of statin therapy.

From three nationwide registers maintained by Statistics Denmark and The National Board of Health, we retrieved historical data on demographics, dispensed cardiovascular drugs (1995-2005), and inpatient-outpatient information (1977-2005). Records at the individual level were linked by means of a unique encrypted person identifier ${ }^{31}$. The linkage allows authorized researchers to follow individuals in multiple individual-level registries hosted in Statistics Denmark through secured Internet access.

All Danish residents, as of January 1, 1996, were identified in the demographic registry $(N=5,114,040)$ and followed until December 31, 2005. The registry contains information on gender, year of birth, and date of death or emigration. To categorize cohort members as incident or prevalent drug users, we used a 1-year run-in period ${ }^{32}$, and accordingly, persons not fully observable during 1995 were excluded. Furthermore, to avoid misclassification of disease history, people who immigrated within 10years before the observation period were excluded, resulting in a final cohort of 4,998,578 members. Analyses were each year limited to individuals older than 20 years. 


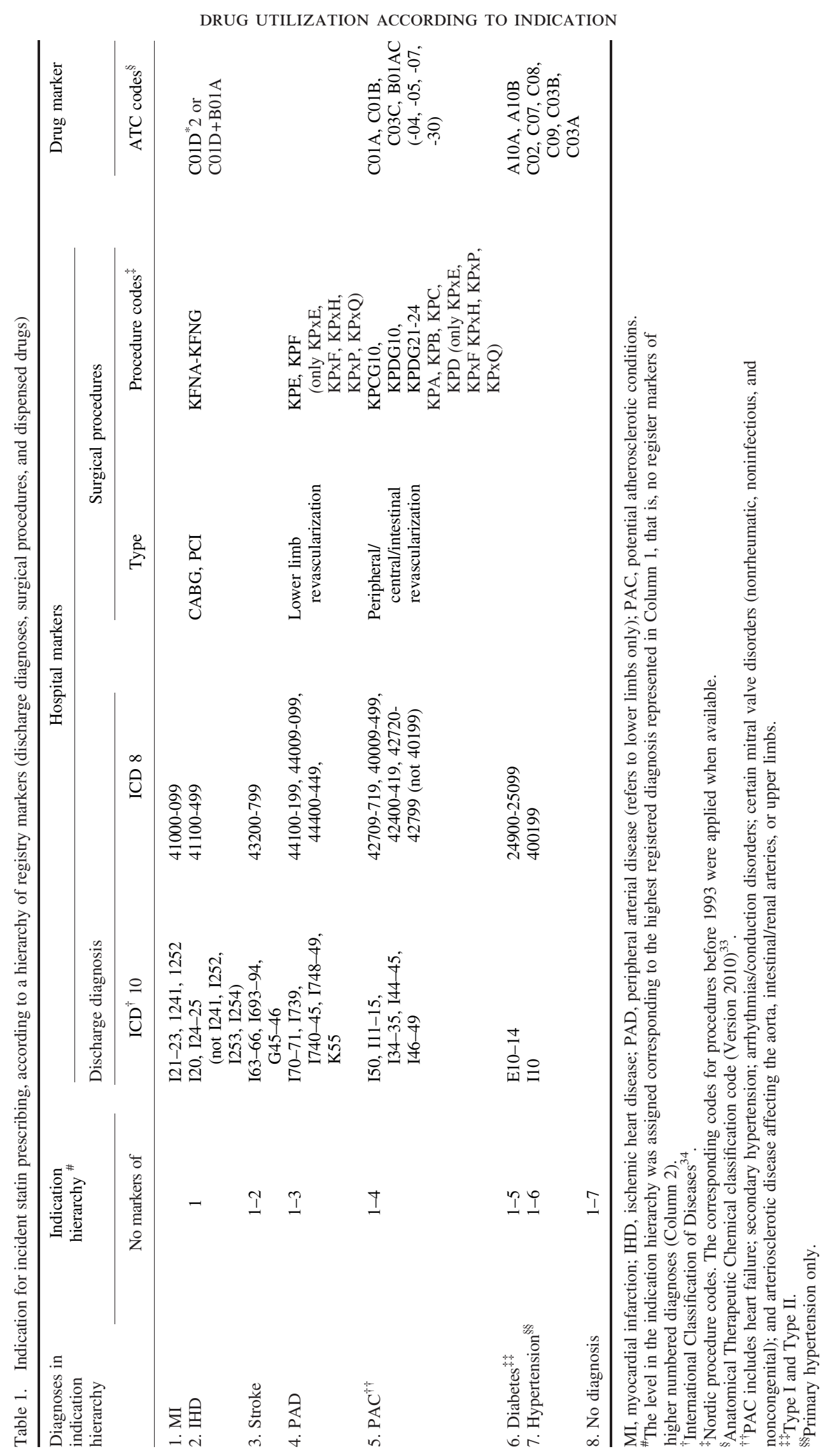

Copyright (C) 2011 John Wiley \& Sons, Ltd. 
The Danish National Prescription Registry (DNPR) contains full information since 1995 on all out-ofhospital purchases of prescription drugs at Danish pharmacies, including prescriptions for nursing home residents ${ }^{19}$. Records include the person identifier, date of dispensing, the Anatomical Therapeutic Chemical (ATC) classification code of the dispensed drug ${ }^{33}$, and the amount in defined daily doses (DDDs). The records do not contain information on prescribed dose or on the indication. From the DNPR, we retrieved information on dispensed cardiovascular drugs (ATC groups C and B01) and antidiabetics (A10).

The Danish National Patient Registry contains information on all patients discharged from non-psychiatric hospitals since 1977. Records include the admission and discharge dates, discharge diagnoses according to the International Classification of Diseases (ICD), 8th revision until 1993 and 10th revision thereafter ${ }^{34}$, along with codes for diagnostic and surgical procedures. We included both primary and secondary diagnoses for admitted patients (discharge date) and patients in ambulant care (admission date) but did not include emergency room visits.

Each cohort member was assigned to the highest possible level in the indication hierarchy corresponding to current diagnosis or drug register markers (Table 1). The first date with a register marker of one of the cardiovascular conditions defines an individual's transition into the corresponding indication level. For example, regardless of any other register markers, individuals with prior MI will be assigned to the highest level, i.e. MI is assumed to be the indication for initiating statin therapy. The IHD level is assigned to individuals without MI, but with either hospital or drug markers indicating IHD (Table 1). Two consecutive dispensing of nitrates (C01DA) or one dispensing of nitrates followed by dispensing of aspirin (acetylsalicylic acid, B01AC06) during a 12-month period were regarded as drug markers of IHD ${ }^{12,35,36}$. To avoid immortal time bias, the last of the two dispensing dates was applied as the IHD transition date. The levels corresponding to stroke or PAD were used only when higher level markers were not present.

Potential atherosclerotic conditions were defined as a range of cardiovascular conditions such as heart failure, secondary hypertension, arteriosclerosis of the aorta, renal or intestinal arteries or upper limbs, certain mitral valve disorders (i.e., nonrheumatic, noninfectious, and noncongenital), and arrhythmias. At this level, we also included drug markers (digitalis glycosides and loop diuretics). For diabetes, we included as markers both dispensed antidiabetics and diabetes diagnoses. As markers for primary hypertension, we applied all potential antihypertensive drugs, the most important groups being beta-blockers, thiazide diuretics, calcium channel blockers, ACE inhibitors, and angiotensin II receptor antagonists. While individuals were categorized as having primary hypertension after the first dispensing of an antihypertensive - provided no markers of higher CVD levels, no diagnosis was assigned to individuals without any of the applied register markers. Individuals at these two lower levels in the hierarchy are referred to as asymptomatic individuals.

The dataset for pharmacoepidemiologic analyses was created by combining individual-level information as to statin dispensing dates with the time sequence of indication transitions. By this means, indication-specific person-time at risk of initiating or discontinuing statin therapy can be calculated.

Prevalent statin users were defined as individuals who by January 1 (index date) had purchased a statin prescription at least once during the preceding 365 days. Incident statin users were defined as non-users with the first statin dispensing between the index date and the next 365 days. While the indication for initiating statin therapy was assumed to correspond to the indication level at the date of first dispensing, the indication for continued statin treatment among prevalent users was assumed to correspond to the level attained in the hierarchy as of the index date of the current year. Thus, the indication could change after the therapy was initiated.

The treatment prevalence proportion was calculated as the number of prevalent statin users at the beginning of a calendar year relative to the total number of individuals at that time. The treatment incidence rate was calculated as the number of incident statin users during a year relative to person-time at risk (censoring at death, emigration, or transition to a higher indication level). We calculated these measures both overall and stratified by calendar year, indication, gender, and age groups.

All analyses were performed using Stata Release 11.1 (StataCorp, College Station, TX, USA). Access to data was provided and secured through collaboration between the University of Copenhagen and Statistics Denmark. Register-based studies in Denmark do not require approval by an ethics board.

\section{RESULTS}

As an illustration of the method, we present statin treatment prevalence and incidence as of 2005, according to indication, gender, and age. 
DRUG UTILIZATION ACCORDING TO INDICATION

Table 2 indicates that the overall statin treatment prevalence was 62/1000 inhabitants (aged 20+ years), corresponding to 237,336 prevalent users. Treatment prevalence was generally higher among men than women (69/1000 vs. 56/1000), with the age-specific treatment prevalence peaking for both genders at the age of 65-74 years. The relative gender difference was most pronounced at younger ages. Whereas women aged $75+$ years accounted for $22.7 \%$ of the female statin users, the same figure was $15.8 \%$ among male users.

Treatment prevalence was highest among individuals with MI $(649 / 1000$ v. 563/1000 in men and women, respectively) and tended to be higher among men with indications in the upper part of the hierarchy, but it was higher among women (especially the older persons) in the lower part of the hierarchy. Whereas MI patients accounted for $29.2 \%$ of the male statin users, the figure was $14.2 \%$ among women. For primary hypertension, in contrast, the figures were $12.4 \%$ and $21.1 \%$ respectively.

The pattern of treatment incidence (Table 3) follows roughly that of treatment prevalence. The highest incidence rate is found among individuals with MI, followed by diabetes, stroke, PAD, and IHD. While the incidence of statin therapy in patients with MI and IHD is highest among men, the opposite is the case in elderly with diabetes, hypertension and those with no diagnosis. Incidence generally decreases above the age of 75 years.

Table 2. Statin treatment prevalence according to indication, gender, and age: number of prevalent statin users and statin treatment prevalence by January 1, 2005

\begin{tabular}{|c|c|c|c|c|c|c|c|c|c|c|c|c|}
\hline \multirow[t]{2}{*}{ Gender } & \multirow{2}{*}{$\begin{array}{l}\text { Age, } \\
\text { years }\end{array}$} & \multirow{2}{*}{\multicolumn{2}{|c|}{$N(\%)^{*}$}} & \multicolumn{9}{|c|}{ Treatment prevalence (per 1000 persons) according to indication $\dagger$, gender, and age } \\
\hline & & & & All & MI & IHD & Stroke & PAD & PAC & Diabetes & Hypertension & Risk \\
\hline \multirow{7}{*}{ Male } & $20-39$ & 2,390 & (1.9) & 4 & 548 & 142 & 95 & 66 & 25 & 101 & 15 & 1 \\
\hline & $40-54$ & 23,190 & (18.0) & 43 & 751 & 354 & 309 & 310 & 102 & 307 & 77 & 10 \\
\hline & $55-64$ & 43,458 & (33.7) & 126 & 760 & 480 & 373 & 357 & 158 & 390 & 116 & 20 \\
\hline & $65-74$ & 39,391 & (30.6) & 196 & 711 & 481 & 346 & 339 & 155 & 370 & 112 & 24 \\
\hline & $75-84$ & 18,963 & (14.7) & 169 & 531 & 309 & 202 & 216 & 79 & 260 & 60 & 13 \\
\hline & +85 & 1,384 & (1.1) & 48 & 194 & 60 & 57 & 60 & 13 & 48 & 13 & 2 \\
\hline & All $^{*}$ & \multicolumn{2}{|c|}{128,776} & 69 & $649(29.2)$ & $386(19.9)$ & $285(9.3)$ & $296(2.5)$ & $111(7.4)$ & $312(10.2)$ & $86(12.4)$ & $9(9.1)$ \\
\hline \multirow{7}{*}{ Female } & $20-39$ & 1,520 & (1.4) & 3 & 490 & 104 & 88 & 64 & 17 & 62 & 4 & 1 \\
\hline & $40-54$ & 13,840 & (12.7) & 26 & 709 & 274 & 238 & 306 & 57 & 246 & 34 & 6 \\
\hline & $55-64$ & 32,469 & (29.9) & 93 & 737 & 393 & 377 & 404 & 129 & 439 & 97 & 23 \\
\hline & $65-74$ & 36,196 & (33.3) & 161 & 720 & 426 & 370 & 392 & 159 & 450 & 130 & 36 \\
\hline & $75-84$ & 22,113 & (20.4) & 134 & 538 & 257 & 220 & 262 & 89 & 301 & 74 & 20 \\
\hline & +85 & 2,488 & (2.3) & 35 & 167 & 48 & 59 & 72 & 15 & 64 & 13 & 3 \\
\hline & $\mathrm{All}^{*}$ & \multicolumn{2}{|c|}{108,590} & 56 & $562(14.2)$ & $281(17.8)$ & 252 (9.9) & $310(3.3)$ & $91(13.2)$ & $301(9.9)$ & $66(21.1)$ & $9(10.6)$ \\
\hline Both & All* & \multicolumn{2}{|c|}{237,366} & 62 & $621(22.3)$ & $332(19.0)$ & 269 (9.6) & $303(2.9)$ & $98(10.0)$ & $307(10.1)$ & $73(16.4)$ & $9(9.8)$ \\
\hline
\end{tabular}

*The distribution $(\%)$ of prevalent statin users according to age or indication.

${ }^{\dagger}$ See Table 1 for definitions and abbreviations.

Table 3. Incidence of statin use in Denmark during 2005: treatment incidence according to indication, gender, and age

\begin{tabular}{|c|c|c|c|c|c|c|c|c|c|}
\hline \multirow[t]{2}{*}{ Gender } & \multirow{2}{*}{$\begin{array}{l}\text { Age, } \\
\text { years }\end{array}$} & \multicolumn{8}{|c|}{ Treatment incidence (per 1000 person-years at risk) according to indication*, gender, and age } \\
\hline & & MI & IHD & Stroke & PAD & $\mathrm{PAC}$ & Diabetes & Hypertension & No diagnosis \\
\hline \multirow{6}{*}{ Male } & $20-39$ & 260 & 36 & 55 & 21 & 20 & 62 & 9 & 1 \\
\hline & $40-54$ & 467 & 101 & 155 & 92 & 48 & 166 & 40 & 5 \\
\hline & $55-64$ & 429 & 135 & 190 & 137 & 68 & 194 & 56 & 10 \\
\hline & $65-74$ & 357 & 136 & 170 & 134 & 65 & 180 & 57 & 11 \\
\hline & $75-84$ & 209 & 90 & 106 & 91 & 35 & 114 & 30 & 7 \\
\hline & +85 & 87 & 27 & 43 & 27 & 11 & 34 & 9 & 1 \\
\hline \multirow{6}{*}{ Female } & $20-39$ & 191 & 32 & 38 & 8 & 8 & 36 & 2 & 1 \\
\hline & $40-54$ & 334 & 77 & 114 & 76 & 27 & 122 & 19 & 3 \\
\hline & $55-64$ & 338 & 107 & 154 & 129 & 53 & 225 & 50 & 12 \\
\hline & $65-74$ & 343 & 115 & 174 & 142 & 59 & 223 & 61 & 17 \\
\hline & $75-84$ & 214 & 74 & 107 & 97 & 37 & 131 & 36 & 11 \\
\hline & +85 & 73 & 20 & 32 & 29 & 7 & 39 & 8 & 3 \\
\hline
\end{tabular}

*See Table 1 for definitions and abbreviations. 
Figure 1 depicts the percentage distribution according to indication among incident statin users during 2005. Among women, primary hypertension is the most frequent indication. Almost 50\% of all incident female users are asymptomatic (hypertension or no diagnosis), whereas MI accounted for less than $10 \%$. Among men, asymptomatic individuals accounted for $35 \%$; MI, for $15 \%$.

Figure 2 shows the percentage distribution of incident statin users according to age and indication stratified by gender. For both genders, the share of incident users with indications at the highest level of the hierarchy increased with age, and for all age groups, this share was highest among men. The two indications highest in the hierarchy (MI and IHD) accounted for $28 \%$ of incident prescribing among men aged 55-64 years and 14\% among women. The corresponding shares were $50 \%$ and $35 \%$ among individuals aged 75-84 years. Conversely, the two indications lowest in the hierarchy (no diagnosis and hypertension) accounted for $40 \%$ of incident prescribing among men aged 55-64 years and 55\% among women. Among individuals aged 75-84 years, the corresponding shares were $15 \%$ and $28 \%$.

\section{DISCUSSION}

\section{Method and principal findings}

Applying register-based information, we developed a pharmacoepidemiologic method for drug utilization analyses according to indication, gender, and age. The method is based on a hierarchy of mutually exclusive indications and allows for in-depth drug utilization analyses of treatment prevalence and incidence according to indication.

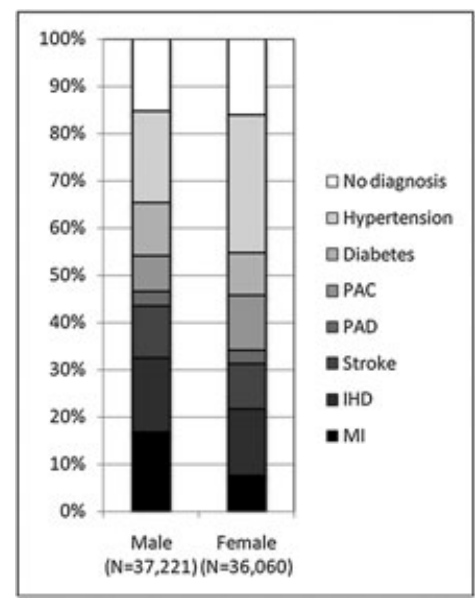

Figure 1. Number of incident statin users by gender during 2005: percentage distribution according to indication. Cf. Table 1 for abbreviations of the diagnoses in the indication hierarchy
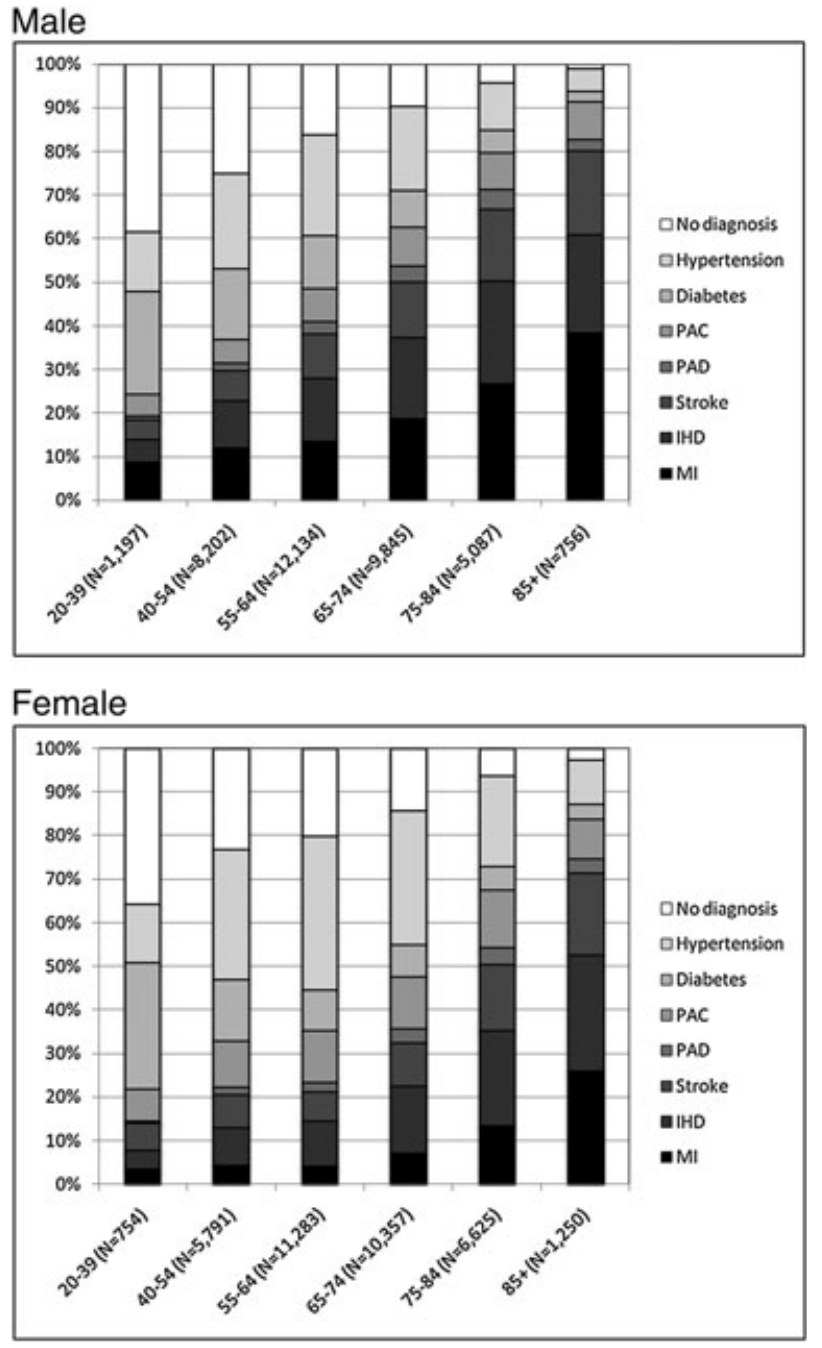

Figure 2. Number of incident statin users during 2005 stratified by gender. Percentage distribution according to age and indication. Cf. Table 1 for abbreviations of the diagnoses in the indication hierarchy

Analyses of statin utilization in 2005 revealed that treatment prevalence was highest among individuals with MI and tended to be higher among men with indications in the upper part of the hierarchy, but it was higher among women (especially the elderly) in the lower part of the hierarchy. Treatment incidence rates followed roughly the same pattern. The share of incident users in the upper part of the hierarchy increased with age.

\section{Strengths and limitations}

Because guidelines are applied as clinical prescribing recommendations, we consider it a strength that our indication hierarchy is based on the recommendations described in the guidelines. The hierarchy implicitly prioritizes among indications such that the indication for statin therapy for an individual with MI will be 
MI-irrespective of eventual subsequent conditions that might have triggered the prescribing. Alternatively, we could have applied an approach where the indication level was determined by a combination of all CVD markers and risk factors. Although this is likely to be too complex for exploring drug utilization patterns, it may be fruitful in multivariate analyses of treatment in different patient groups according to co-morbidity.

The challenge was to identify credible register markers for the conditions reflecting the recommended statin indications. By systematically identifying all potential register markers (in-hospital and drug markers), we were able to establish a hierarchy of eight mutually exclusive indication groups, including a gray-zone group with PAC and a no diagnosis group. Without the gray-zone group, subjects with more-or-less obvious atherosclerotic conditions would have been misclassified downward in the hierarchy-eventually to the no diagnosis group. Owing to the strong evidence for statin therapy among individuals with PAD ${ }^{25}$, we opted to restrict this subgroup to PAD only-leaving, for example, aorta aneurism in the gray-zone group.

The main limitation of the study is the lack of information on familial hypercholesterolemia, microalbuminuria (statin indication criterion for Type I diabetics), and low ankle blood pressure (indicating PAD). Consequently, CVD-free statin users among the approximately 10,000 individuals with familial hypercholesterolemia in Denmark ${ }^{37}$ are classified as having no diagnosis. We also opted not to distinguish between Type I and Type II diabetes.

To reduce the risk of misclassification, we traced hospital information on CVD since the beginning of registration in 1977. This is a strength of this study, representing a considerably longer period of observation than that applied in similar studies ${ }^{11-13}$. We evaluated the impact of applying shorter diagnosis run-in periods to capture surviving individuals with prior MI by means of registered hospital MI diagnoses. Although only $60 \%$ of the MI patients would be captured applying a run-in period of 3 years, $74 \%$ and $94 \%$ would be captured with run-in periods of 5 and 10 years, respectively. With a run-in period of 3 years, the distribution of incident statin users, shown in Figure 1, would shift towards "lighter" diagnoses: about seven percentage points fewer statin-users would be categorized as initiating therapy on MI/ IHD indication, and a higher percentage would belong to the hypertension and PAC categories. We thus applied a diagnosis run-in window of at least 10 years for immigrants to minimize the bias introduced when comparing statin indication over time.
In line with a study from British Columbia ${ }^{13}$, we found a tendency for relatively high treatment prevalence among women and the older persons "without heart disease" - applying their rather broad categories. However, our analysis demonstrates a more nuanced picture. In contrast to a study from Ireland exclusively using dispensing information as disease markers ${ }^{12}$, we applied both in-hospital and dispensing information as register markers, making it possible to distinguish between primary and secondary hypertension. Furthermore, drug information was in our study applied to identify patients exclusively treated in primary care. To categorize an individual as an IHD patient, we required two consecutive dispensings of nitrates (or nitrate followed by aspirin), applying the last of the two dates as the transition date to IHD to avoid immortal time bias. Yet, this may lead to misclassification of some incident users. As this presumably small group will be correctly categorized as prevalent users the following year, we consider this latter bias less serious.

Antihypertensives may also be used for other indications, for example, ACE inhibitors and angiotensin II antagonists for heart failure, beta-blockers, and calcium channel blockers for angina pectoris or supraventricular tachycardia. These indications belong to the IHD or PAC categories, but we assume that the majority of heart patients (not kidney patients) would be captured by inpatient or outpatient diagnoses. Thus, we used thiazides as markers of primary hypertension and loop diuretics as markers of PAC, which corresponds to Danish guidelines recommending loop diuretics in hypertension patients with heart failure or nephropathy but not in uncomplicated hypertension. Users of beta-blockers for migraine or thiazides for edema, however, may be misclassified as having hypertension. Thus, further validation of the drug markers for hypertension based on information from primary care is needed.

The stringent pharmacoepidemiologic approach enabled us to calculate the precise number of statin users and the corresponding denominators, stratified by indication. This allows for unbiased calculations of treatment incidence and prevalence. Raymond and colleagues applied a register-based statin indication hierarchy ${ }^{11}$-similar to ours as to the medical conditions, but not regarding the pharmacoepidemiologic measures. Applying the whole population as denominator, incidence and prevalence according to indication tend to be underestimated and time trends will be hard to detect. Without day-to-day information about new CVD diagnoses, incident statin users with 
a recent CVD diagnosis will be classified corresponding to the diagnosis level as of the index date (e.g., January 1).

\section{Applications of the method}

The person-time applied for calculations of incidence rate can also be used to estimate treatment discontinuation rates according to indication, defining for instance discontinuation as 365 days since last prescription ${ }^{20}$. As adherence (persistence) to therapy is an important aspect of drug treatment ${ }^{38}$, the method also applies for measuring adherence according to indication. In addition, the method can be used to consider the epidemiology of the disease, that is, the incidence and prevalence of diseases in the hierarchy, and thus also to calculate the proportion of nevertreated patients.

The indication hierarchy may be elaborated for other drug categories (e.g., antidepressants and bisphosphonates) to study a potential indication creep. A hierarchy for antidepressants could be based on psychiatric discharge diagnoses, co-morbidities, and prescriber categories (specialist or general practitioner). For bisphosphonates, the hierarchy could be based on indication markers such as osteoporosisrelated fractures, bone mineral density scans, treatment with systemic corticosteroids, bone metastases, and so forth.

Postmarketing surveillance studies tend to focus on potential adverse effects without assessing a riskto-benefit trade-off, which is likely to depend on the indication. Analyses combining the indication hierarchy and a risk-to-benefit ratio may help to make treatment recommendations more specific. This could be done both for drugs used symptomatically with potentially life-threatening adverse effects, like COX2 inhibitors, and for preventive drugs, such as statins, with less severe adverse effects. In the former case, diagnoses of rheumatic diseases and other conditions with chronic pain could be combined with markers of the risk of adverse effects, for example, previous peptic ulcers.

Cost-effectiveness analyses of drug therapies are increasingly applied in decision making, but the result depends to a large extent on actual indication patterns and adherence to therapy. The number of prevalent users, the volume of use in DDDs, and drug expenditures could be analyzed using the indication hierarchy, shedding light on cost-effectiveness in clinical practice. Finally, we believe that the method will be useful in cross-national comparisons of drug utilization.

\section{Conclusion}

The proposed indication hierarchy provided insight into prescription indication patterns as to statin utilization in 2005. The method can be implemented for other drug categories and is considered useful for studying trends in drug utilization, differential drug adherence, and cross-national comparisons.

\section{CONFLICT OF INTEREST}

The authors declare no conflict of interests.

\section{KEY POINTS}

- The utilization of many drug categories has increased dramatically during the last decades, posing an increasing burden on health care resources. Yet, it is unknown to what extent the increasing utilization is due to changing prescribing indications - or an indication creep.

- We developed a pharmacoepidemiologic method to analyze drug utilization according to indication, age, gender, and calendar year. Applying a hierarchy of register-based markers of recommended prescribing indications, the method was demonstrated on statin utilization in 2005.

- The analysis revealed that statin treatment prevalence and incidence tended to be higher among men with indications in the upper part of the hierarchy (MI or IHD), but it was higher among women in the lower part of the hierarchy (primary hypertension or no-register-based diagnosis).

- Although the actual indication hierarchy will be useful for studying trends in statin utilization and statin adherence, the method could also be adapted to other drug categories.

\section{ACKNOWLEDGEMENT}

This study was supported by a grant from the Nordea Foundation.

\section{REFERENCES}

1. Randomised trial of cholesterol lowering in 4444 patients with coronary heart disease: the Scandinavian Simvastatin Survival Study (4S). Lancet 1994; 344(8934): 1383-1389.

2. Ong HT. The statin studies: from targeting hypercholesterolaemia to targeting the high-risk patient. QJM 2005; 98(8): 599-614.

3. Shepherd J. The West of Scotland Coronary Prevention Study: a trial of cholesterol reduction in Scottish men. Am J Cardiol 1995; 76(9): 113C-117C.

4. Sacks FM, Pfeffer MA, Moye LA, et al. The effect of pravastatin on coronary events after myocardial infarction in patients with average cholesterol levels. 


\section{DRUG UTILIZATION ACCORDING TO INDICATION}

Cholesterol and Recurrent Events Trial investigators. N Engl J Med 1996; 335(14): 1001-1009.

5. Prevention of cardiovascular events and death with pravastatin in patients with coronary heart disease and a broad range of initial cholesterol levels. The Long-Term Intervention with Pravastatin in Ischaemic Disease (LIPID) Study Group. N Engl J Med 1998; 339(19): 1349-1357.

6. MRC/BHF Heart Protection Study of cholesterol lowering with simvastatin in 20,536 high-risk individuals: a randomised placebo-controlled trial. Lancet 2002; 360(9326): 7-22.

7. Sever PS, Dahlof B, Poulter NR, et al. Prevention of coronary and stroke events with atorvastatin in hypertensive patients who have average or lower-than-average cholesterol concentrations, in the Anglo-Scandinavian Cardiac Outcomes Trial-Lipid Lowering Arm (ASCOT-LLA): a multicentre randomised controlled trial. Lancet 2003; 361(9364): 1149-1158.

8. Colhoun HM, Betteridge DJ, Durrington PN, et al. Primary prevention of cardiovascular disease with atorvastatin in type 2 diabetes in the Collaborative Atorvastatin Diabetes Study (CARDS): multicentre randomised placebo-controlled trial. Lancet 2004; 364(9435): 685-696.

9. Downs JR, Clearfield M, Weis S, et al. Primary prevention of acute coronary events with lovastatin in men and women with average cholesterol levels: results of AFCAPS/TexCAPS. Air Force/Texas Coronary Atherosclerosis Prevention Study. JAMA 1998; 279(20): 1615-1622.

10. Shepherd J, Blauw GJ, Murphy MB, et al. Pravastatin in elderly individuals at risk of vascular disease (PROSPER): a randomised controlled trial. Lancet 2002; 360(9346): 1623-1630.

11. Raymond CB, Morgan SG, Katz A, et al. A population-based analysis of statin utilization in British Columbia. Clin Ther 2007; 29(9): 2107-2119.

12. Teeling M, Bennett K, Feely J. The influence of guidelines on the use of statins: analysis of prescribing trends 1998-2002. Br J Clin Pharmacol 2005; 59(2): 227-232.

13. Savoie I, Kazanjian A. Utilization of lipid-lowering drugs in men and women. a reflection of the research evidence? J Clin Epidemiol 2002; 55(1): 95-101.

14. Ruokoniemi P, Helin-Salmivaara A, Klaukka T, et al. Shift of statin use towards the elderly in 1995-2005: a nation-wide register study in Finland. Br J Clin Pharmacol 2008; 66(3): 405-410.

15. DeWilde S, Carey IM, Bremner SA, et al. Evolution of statin prescribing 1994-2001: a case of agism but not of sexism? Heart 2003; 89(4): 417-421.

16. Abookire SA, Karson AS, Fiskio J, et al. Use and monitoring of "statin" lipidlowering drugs compared with guidelines. Arch Intern Med 2001; 161(1): 53-58.

17. Selmer R, Sakshaug S, Skurtveit S, et al. Statin treatment in a cohort of 20212 men and women in Norway according to cardiovascular risk factors and level of education. Br J Clin Pharmacol 2009; 67(3): 355-362.

18. Thygesen LC, Daasnes C, Thaulow I, et al. Introduction to Danish nation-wide registers on health and social issues - structure, access, legislation and archiving. Scand J Public Health 2011; 39(Supple 7): 12-16.

19. Kildemoes HW, Sorensen HT, Hallas J. The Danish National Prescription Registry. Scand J Public Health 2011; 39(Supple 7): 38-41.

20. Kildemoes HW, Stovring H, Andersen M. Driving forces behind increasing cardiovascular drug utilization: a dynamic pharmacoepidemiological model. $\mathrm{Br} J$ Clin Pharmacol 2008; 66(6): 885-895.

21. Pyorala K, De Backer G, Graham I, et al. Prevention of coronary heart disease in clinical practice. Recommendations of the Task Force of the European Society of Cardiology, European Atherosclerosis Society and European Society of Hypertension. Eur Heart J 1994; 15(10): 1300-1331.

22. De Backer G, Ambrosioni E, Borch-Johnsen K, et al. European guidelines on cardiovascular disease prevention in clinical practice. Third Joint Task Force of
European and Other Societies on Cardiovascular Disease Prevention in Clinical Practice. Eur Heart J 2003; 24(17): 1601-1610.

23. Graham I, Atar D, Borch-Johnsen K, et al. European guidelines on cardiovascular disease prevention in clinical practice: executive summary: Fourth Joint Task Force of the European Society of Cardiology and Other Societies on Cardiovascular Disease Prevention in Clinical Practice (Constituted by representatives of nine societies and by invited experts). Eur Heart $J$ 2007; 28(19): 2375-2414.

24. Wood D, De Backer G, Faergeman O, et al. Prevention of coronary heart disease in clinical practice: recommendations of the Second Joint Task Force of European and other Societies on Coronary Prevention. Atherosclerosis 1998; 140 (2): 199-270.

25. Hirsch AT, Haskal ZJ, Hertzer NR, et al. ACC/AHA Guidelines for the Management of Patients with Peripheral Arterial Disease (lower extremity, renal, mesenteric, and abdominal aortic). J Vasc Interv Radiol 2006; 17(9): $1383-1397$.

26. Christensen B, Heebøll-Nielsen N, Madsen L, et al. Forebyggelse af iskæmisk hjertesygdom i almen praksis - med særligt henblik på dyslipidæmi: En klinisk vejledning [Prevention of ishaemic heart disease in general practice - with special reference to dyslipidemia: A clinical guidance]. Danish Medical Association (DSAM): Aalborg, 1998.

27. Christensen B, Færgemann O, Heebøll-Nielsen N, et al. Forebyggelse af iskæmisk hjerte-kar-sygdom i almen praksis. En klinisk vejledning [Prevention of ischemic cardiovascular disease in almen practice. A clinical guidance.]. Danish Medical Association (DSAM): Denmark, 2007.

28. Thomsen T, Christensen B, Hildebrant $\mathrm{P}$, et al. Kliniske retningslinier for forebyggelse af kardiovaskulær sygdom i Danmark [Clinical guidance for prevention of cardiovasular disease in Denmark]. The European Society of Cardiology: Denmark, 2004.

29. Færgemann O, Christensen B, Hansen HS, et al. Sekundær og primær forebyg gelse af koronar hjertesygdom med særligt henblik på dyslipidæmi [Secondary and primary prevention of coronar heart disease ved speciel reference to dydlipedemia]. Danish Society of Cardiology: Denmark, 2000.

30. Conroy RM, Pyorala K, Fitzgerald AP, et al. Estimation of ten-year risk of fatal cardiovascular disease in Europe: the SCORE project. Eur Heart J 2003; 24(11) 987-1003.

31. Petersen CB. The Danish Civil Registration System. Scand J Public Health 2011; Suppl (in press).

32. Hallas J, Stovring $\mathrm{H}$. Templates for analysis of individual-level prescription data Basic Clin Pharmacol Toxicol 2006; 98(3): 260-265.

33. WHO Collaborating centre for Drug Statistics Methodology, Guidelines for ATC classification and DDD assignment, 2010. Norwegian Institute of Public Health: Oslo, 2009.

34. Andersen TF, Madsen M, Jorgensen J, et al. The Danish National Hospital Reg ister. A valuable source of data for modern health sciences. Dan Med Bull 1999 46(3): 263-268.

35. Cannon PJ, Connell PA, Stockley IH, et al. Prevalence of angina as assessed by a survey of prescriptions for nitrates. Lancet 1988; 1(8592): 979-981.

36. Gray J, Majeed A, Kerry S, et al. Identifying patients with ischaemic heart disease in general practice: cross sectional study of paper and computerised medical records. BMJ 2000; 321(7260): 548-550.

37. Jensen HK. The molecular genetic basis and diagnosis of familial hypercholesterolemia in Denmark. Dan Med Bull 2002; 49(4): 318-345.

38. Larsen J, Vaccheri A, Andersen M, et al. Lack of adherence to lipid-lowering drug treatment. A comparison of utilization patterns in defined populations in Funen, Denmark and Bologna, Italy. Br J Clin Pharmacol 2000; 49(5): $463-471$. 